\title{
Markov chain beam randomization: a study of the impact of PLANCK beam measurement errors on cosmological parameter estimation
}

\author{
G. Rocha ${ }^{1,2}$, L. Pagano ${ }^{3}$, K. M. Górski ${ }^{1,2,4}$, K. M. Huffenberger ${ }^{5}$, C. R. Lawrence $^{1}$, and A. E. Lange ${ }^{2}$ \\ 1 Jet Propulsion Laboratory, California Institute of Technology, 4800 Oak Grove Drive, Pasadena CA 91109, USA \\ 2 California Institute of Technology, Pasadena CA 91125, USA \\ 3 Physics Department and sezione INFN, University of Rome "La Sapienza", Ple Aldo Moro 2, 00185 Rome, Italy \\ 4 Warsaw University Observatory, Aleje Ujazdowskie 4, 00478 Warszawa, Poland \\ ${ }^{5}$ Department of Physics, University of Miami, 1320 Campo Sano Avenue, Coral Gables, FL 33124, USA
}

Received 31 July 2009 / Accepted 13 January 2010

\begin{abstract}
We introduce a new method to propagate uncertainties in the beam shapes used to measure the cosmic microwave background to cosmological parameters determined from those measurements. The method, called markov chain beam randomization (MCBR), randomly samples from a set of templates or functions that describe the beam uncertainties. The method is much faster than direct numerical integration over systematic "nuisance" parameters, and is not restricted to simple, idealized cases as is analytic marginalization. It does not assume the data are normally distributed, and does not require Gaussian priors on the specific systematic uncertainties. We show that MCBR properly accounts for and provides the marginalized errors of the parameters. The method can be generalized and used to propagate any systematic uncertainties for which a set of templates is available. We apply the method to the Planck satellite, and consider future experiments. Beam measurement errors should have a small effect on cosmological parameters as long as the beam fitting is performed after removal of $1 / f$ noise.
\end{abstract}

Key words. cosmic microwave background - cosmology: observations - methods: data analysis

\section{Introduction}

Observations of the cosmic microwave background (CMB) can be interpreted only in light of a detailed knowledge of the angular response of the instrument to radiation, i.e., the shapes of the "beams". It is almost always the case that the beams from single-aperture telescopes (but not interferometers) can be approximated as two-dimensional Gaussians. It is never the case that a gaussian approximation provides an adequate description of the beams of an experiment that measures the CMB with high signal-to-noise ratio. If the beams were known perfectly, their effects on the data could be calculated perfectly, if painfully. Unfortunately, beams are never known perfectly, and among the outstanding issues for any CMB experiment are how to optimize the beams in the first place, and how to control and account for beam uncertainties in the data analysis.

The effects of beam uncertainties can be analyzed in maps, power spectra, and cosmological parameters determined from the data. Each has benefits. Because cosmological parameters are a key product of any experiment, and because they are sensitive to extremely small effects impossible to detect pixel by pixel, they are particularly valuable. Historically, however, calculation of the effects of beam uncertainties on cosmological parameters has been done either analytically, which requires over-simplified beam shapes, or numerically, at great computational cost.

We introduce in this paper a method for calculating the effects of beam uncertainties on cosmological parameters determined from CMB observations that is both fast and flexible. It requires only that beam uncertainties, or for that matter any other systematic effect, can be represented by a set of functions or templates, which could be obtained from Monte Carlo simulations. It does not assume that the data themselves are Gaussiandistributed, or that the uncertainties have Gaussian priors.

In Sect. 2 we describe the method, called markov chain beam randomization or MCBR, and we show that the MCBR technique produces correct marginalized errors. Section 3 summarizes the beam fitting procedure developed in a previous paper (Huffenberger et al. 2010). Section 4 describes the implementation of MCBR. In Sect. 5 we apply the method to the Planck experiment, and consider future experiments.

\section{MCBR: markov chain beam randomization}

In the past, marginalization over systematic parameters has been carried out either numerically or analytically (Bridle et al. 2002); both methods are currently implemented in cosmomc (Lewis \& Bridle 2002). Assuming likelihoods are Gaussian one typically has a marginalization of the form:

$L \propto \int \mathrm{d} \alpha P(\alpha) \exp \left[-(\alpha \boldsymbol{v}-\boldsymbol{d})^{\mathrm{T}} \mathbf{N}^{-1}(\alpha \boldsymbol{v}-\boldsymbol{d}) / 2\right]$

where $\boldsymbol{d}$ is the theoretical (predicted) data minus the observed data and $\alpha v$ is an approximate template describing the correction applied to the predicted data due to systematics, $\mathbf{N}$ is the noise 
covariance matrix, and $P(\alpha)$ is the prior. The marginalization is given by:

$-2 \ln L=\boldsymbol{d}^{\mathrm{T}}\left(\mathbf{N}^{-1}-\frac{\mathbf{N}^{-1} \boldsymbol{v} \boldsymbol{v}^{\mathrm{T}} \mathbf{N}^{-1}}{\boldsymbol{v}^{\mathrm{T}} \mathbf{N}^{-1} \boldsymbol{v}}\right) \boldsymbol{d}+\ln \left(\boldsymbol{v}^{\mathrm{T}} \mathbf{N}^{-1} \boldsymbol{v}\right)+\mathrm{c}$

where $\mathrm{c}$ is a constant. If $v$ is independent of the data and parameters then $L \propto \mathrm{e}^{-\chi_{\text {eff }}^{2} / 2}$, with:

$\chi_{\text {eff }}^{2}=\boldsymbol{d}^{\mathrm{T}}\left(\mathbf{N}^{-1}-\frac{\mathbf{N}^{-1} \boldsymbol{v} \boldsymbol{v}^{\mathrm{T}} \mathbf{N}^{-1}}{\boldsymbol{v}^{\mathrm{T}} \mathbf{N}^{-1} \boldsymbol{v}}\right) \boldsymbol{d}=\chi_{\text {best-fit }}^{2}$.

In the case of beam uncertainties, the analytic approach is feasible only if the beams are assumed to be Gaussian. This is not realistic.

It is customary to characterize anisotropies in the Cosmic Microwave Background by their angular power spectrum, $\boldsymbol{C}_{\ell}$ for both temperature and polarization. $\boldsymbol{C}_{\ell}$ is a $3 \times 3$ matrix for $T$ (temperature) and $\mathrm{E}$ or $\mathrm{B}$ (grad-type or curl-type polarization):

$\mathbf{C}_{\ell}=\left(\begin{array}{lll}C_{\ell}^{\mathrm{TT}} & C_{\ell}^{\mathrm{TE}} & C_{\ell}^{\mathrm{TB}} \\ C_{\ell}^{\mathrm{TE}} & C_{\ell}^{\mathrm{EE}} & C_{\ell}^{\mathrm{EB}} \\ C_{\ell}^{\mathrm{TB}} & C_{\ell}^{\mathrm{EB}} & C_{\ell}^{\mathrm{BB}}\end{array}\right)$.

Hereafter, for the sake of simplicity, most equations will refer to the angular power spectrum, $C_{\ell}$, for a single component, say temperature. The telescope beam smooths the anisotropies, supressing power at higher multipoles. We refer to the ratio of the measured power spectrum of the sky and our true power spectrum as the transfer function, $\mathcal{B}_{\ell}=B_{\ell}^{2}$. Here we assume the beam transfer functions are the same for temperature and polarization.

To obtain unbiased estimates of the parameters that characterize the cosmology, we must repair this suppression based on knowledge of the beam. Uncertainties in the beam propagate into uncertainties in the cosmological parameters.

We assume that the beam uncertainties can be described by a set of functions or templates, taken here to be the set of transfer functions obtained by the beam fitting procedure described in Sect. 3. These templates are given in multipole space by:

$\mathcal{B}_{\ell}^{r}=\left(B_{\ell}^{r}\right)^{2}=\left(B_{\ell} \times r_{\ell}\right)^{2}$

where the ratios $r_{\ell}$ represent the possible deviations from the true fiducial beam. We choose the beam transfer function randomly from the set of $N$ simulations (here $N=1280$ ) for each step of the markov chain Monte Carlo when probing the cosmological parameters space. This means that at each step of the chain the theoretical power spectrum, $\boldsymbol{C}_{\ell}$, is multiplied by the randomly chosen beam, $\mathcal{B}_{\ell}^{r}$. We assume all transfer functions in the set are equally probable.

To estimate constraints on cosmological parameters, we need to compare the model with the data via a chosen Likelihood and an algorithm to sample cosmological parameters. Here we make use of the package cosmomc. To incorporate MCBR we modify cosmomc to enable the usage of a random $\mathcal{B}_{\ell}^{r}$ for each theoretical model generated with CAMB (Lewis et al. 2000) or PICO (Fendt \& Wandelt 2006). This is done by modifying the cmbdata module of the cosmomc code.

We start by creating simulated datasets with noise properties specific to the instrument under consideration, in our case Planck and an example of a future experiment (see Sect. 4). These simulated datasets are given in terms of the angular Power Spectrum $C_{\ell}^{\text {obs: }}$

$C_{\ell}^{\mathrm{obs}}=C_{\ell}^{\mathrm{wmap}} \mathcal{B}_{\ell}+\mathcal{N}_{\ell}$ where $\mathcal{N}_{\ell}$ is the noise power spectrum and $\mathcal{B}_{\ell}=B_{\ell}^{2}$ is the beam transfer function and $C_{\ell}^{\text {wmap }}$ is the $\Lambda$ CDM spectrum bestfitting current WMAP data. In the case of a symmetric Gaussian beam, $B(\boldsymbol{x})=\frac{1}{2 \pi \sigma^{2}} \exp \left\{\frac{-|\boldsymbol{x}|^{2}}{2 \sigma^{2}}\right\}$, so that $B_{\ell}=\mathrm{e}^{-\frac{1}{2} \sigma^{2} \ell^{2}}$. However, the Planck beams are not adequately represented by Gaussians. Instead, we use realistic beams calculated from a full diffraction analysis of the telescope using GRASP9 (Sandri et al. 2002, 2010, in prep.; Maffei et al. 2010, in prep.; Yurchenko et al. Yurchenko et al. 2004).

As our purpose here is to introduce and validate the MCBR method it suffices to assume full-sky coverage. Considerations of realistic complications (such as cut-sky, foregrounds, etc.) is deferred to a future publication. Our purpose here is to establish the relative importance of propagating beam errors to cosmological parameters rather than to make comprehensive predictions for Planck. Hereafter to compare the observed dataset, $\boldsymbol{C}_{\ell}^{\text {obs }}$, with theoretical models we use the exact full-sky likelihood (with $\hat{\boldsymbol{C}}_{\ell}=\boldsymbol{C}_{\ell}^{\mathrm{obs}}$ ) (Bond et al. 2000):

$$
-2 \ln L\left(\hat{\boldsymbol{C}}_{\ell} \mid \boldsymbol{C}_{\ell}\right)=(2 \ell+1)\left(\ln \left|\boldsymbol{C}_{\ell}\right|+\operatorname{Tr}\left(\hat{\boldsymbol{C}}_{\ell} \boldsymbol{C}_{\ell}^{-1}\right)\right),
$$

i.e., the Inverse Wishart distribution for Temperature and Polarization. In cosmomc this distribution is coded in function ChiSqExact (Lewis 2005). We analyse these datasets with a modified version of this function, built to include the MCBR procedure in the code.

The $C_{\ell}$ of the theoretical model is given by:

$\tilde{C}_{\ell}=C_{\ell} \times \mathcal{B}_{\ell}^{r}$

where $\mathcal{B}_{\ell}^{r}$ is the randomly chosen transfer function. To incorporate both the beam and the uniform white noise in the likelihood expression one should replace:

$\hat{C}_{\ell} \rightarrow C_{\ell}^{\mathrm{obs}}$

$C_{\ell} \rightarrow C_{\ell}^{\text {th }} \times \mathcal{B}_{\ell}^{r}+\mathcal{N}_{\ell}$

where $C_{\ell}^{\text {obs }}$ is given by Eq. $6, C_{\ell}^{\text {th }}$ is the theoretical power spectrum computed e.g. by CAMB, and $B_{\ell}^{r}$ is the randomly chosen beam transfer function.

In the MCBR scheme, sampling of the beam templates is equivalent to sampling from the proposal distribution. The Metropolis-Hastings algorithm accepts the move from $\theta_{n}$ to $\theta_{n+1}$ in the Markov chain by evaluating the ratio:

$\frac{P\left(\theta_{n+1}\right) q\left(\theta_{n+1}, \theta_{n}\right)}{P\left(\theta_{n}\right) q\left(\theta_{n}, \theta_{n+1}\right)}$

where $P$ is the posterior distribution we wish to sample from and $q$ is the proposal distribution. We draw the proposal at position $\theta_{n}$ of the parameter space from $q\left(\theta_{n+1}, \theta_{n}\right)$. Here $\theta=\left(\theta_{\mathrm{cp}}, \theta_{\mathrm{b}}\right)$, with $\theta_{\mathrm{cp}}$ the subset of cosmological parameters and $\theta_{\mathrm{b}}$ the beam parameter. The joint proposal density for $\theta$ factors into

$q\left(\theta_{n+1}, \theta_{n}\right)=q_{\mathrm{cp}}\left(\theta_{\mathrm{cp}, n+1}, \theta_{\mathrm{cp}, n}\right) q_{\mathrm{b}}\left(\theta_{\mathrm{b}, n+1}, \theta_{\mathrm{b}, n}\right)$,

where $\theta_{\mathrm{cp}, n+1}$ refers to $\left(\theta_{\mathrm{cp}}\right)_{n+1}$ and $\theta_{\mathrm{b}, n+1}$ to $\left(\theta_{\mathrm{b}}\right)_{n+1}$. Now, we take the $q_{\mathrm{b}}\left(\theta_{\mathrm{b}, n+1}, \theta_{\mathrm{b}, n}\right)$ to be the posterior distribution of the beam parameters given the beam fitting data (in our case the Jupiter beam fitting data (see Sect. 3)), i.e.,

$q_{\mathrm{b}}\left(\theta_{\mathrm{b}, n+1}, \theta_{\mathrm{b}, n}\right)=P_{\mathrm{b}}\left(\theta_{\mathrm{b}, n} \mid\right.$ beamdata $)$.

Furthermore

$P\left(\theta_{n+1}\right)=P_{\mathrm{cp}}\left(\theta_{n+1} \mid\right.$ mapdata $) P_{\mathrm{b}}\left(\theta_{\mathrm{b}, n+1} \mid\right.$ beamdata $)$ 

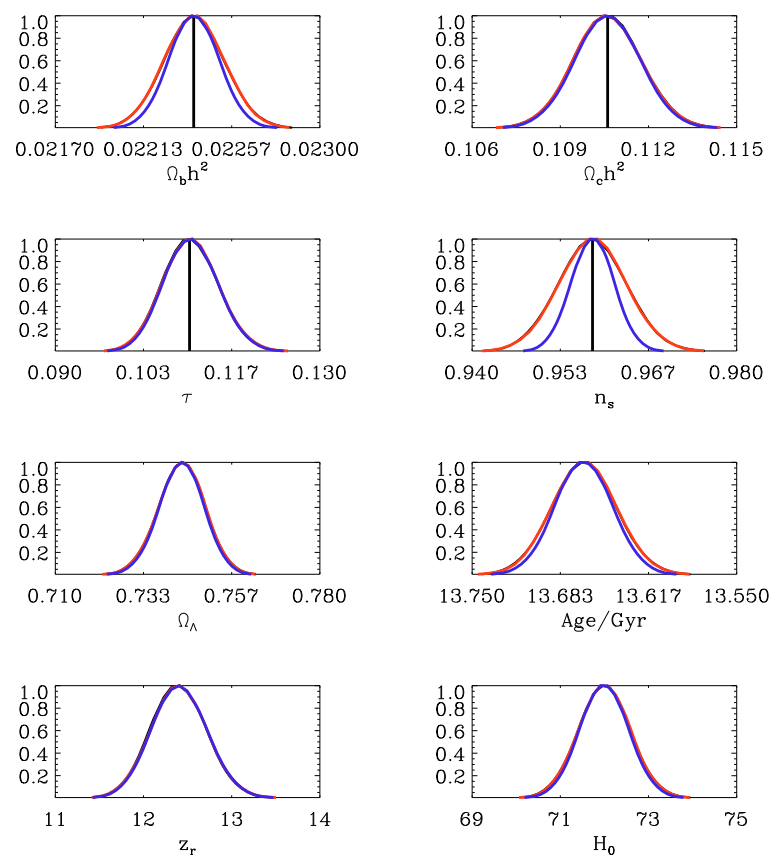
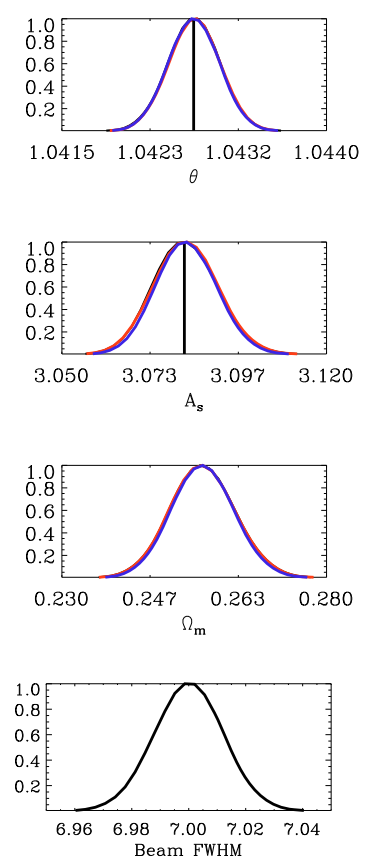

Fig. 1. Marginalized parameter constraints for Planck $143 \mathrm{GHz}$ with 7' beam with $4 \%$ variations, for the analysis with the "true" reference fiducial beam using beamparameter approach (black) and MCBR (red), the blue line is the analysis of the same dataset without including the beam uncertainty. (for instance in our study here $P_{\mathrm{cp}}(\theta \mid$ mapdata) $=$ $L\left(\right.$ mapdata $\left.\mid \theta_{\mathrm{cp}}, \theta_{\mathrm{b}}\right) p_{\mathrm{cp}}\left(\theta_{\mathrm{cp}}\right)$ where $L$ is the Likelihood given in Eq. (7) and $p_{\mathrm{cp}}$ the prior on cosmological parameters). Hence the ratio in Eq. (11) becomes:

$\frac{P_{\mathrm{cp}}\left(\theta_{n+1}\right) q_{\mathrm{cp}}\left(\theta_{\mathrm{cp}, n+1}, \theta_{\mathrm{cp}, n}\right)}{P_{\mathrm{cp}}\left(\theta_{n}\right) q_{\mathrm{cp}}\left(\theta_{\mathrm{cp}, n}, \theta_{\mathrm{cp}, n+1}\right)}$

as $P_{\mathrm{b}}$ and $q_{\mathrm{b}}$ cancel out.

Hence random sampling from the set of beam templates at each step of the Markov chain is equivalent to sampling from a proposal density that, by construction, is identical to the posterior distribution of the beam parameters given the beam fitting data.

To illustrate how the MCBR procedure works, we give here the steps followed in our analysis (see Sect. 5). We start by comparing two cases:

1. cosmomc run with the "true" fiducial beam transfer alone, $\mathcal{B}_{\ell}$;

2. cosmomc run with the MCBR procedure for the set of beam transfer functions, $\mathcal{B}_{\ell}^{r}$, obtained from the beam fitting step.

To this end:

- we generate a simulated data set using as fiducial the "true" beam transfer $\mathcal{B}_{\ell}$;

- we analyse this simulated data set with cosmomc, including in the code just the effect of the $\mathcal{B}_{\ell}$ (i.e. the theoretical $C_{\ell}$ is multiplied by the true beam transfer, $\mathcal{B}_{\ell}$ );

- we analyse this simulated data set with a modified version of cosmomc in which the theoretical $C_{\ell}$ is multiplied by the randomly chosen beam, $\mathcal{B}_{\ell}^{r}$ at each step of the chain - i.e., with in-built MCBR

The MCBR method can be used to propagate any systematic uncertainties that can be characterized by a set of templates. We turn these into multiplicative and additive corrections to the $C_{\ell}$, encode the corrected $C_{\ell}$ into the likelihood, and randomly sample from the set of templates at the markov chain Monte Carlo step of parameter estimation. The data do not have to be normally distributed. Furthermore, unlike analytic marginalization, the method does not require Gaussian priors on the uncertainties.

\subsection{Validation}

To demonstrate that that the MCBR technique gives correct marginalized errors, we compared the results given by MCBR to those from a "brute force" cosmomc calculation in which the beam was taken as another parameter. We did this for three simulated datasets, the first generated using the "true" beam transfer function $\mathcal{B}_{\ell}$, the second and third using beam transfer functions that were chosen to be mildly and extremely far from the true one, respectively. We simplified the test cases by assuming that the beam was a symmetric $7^{\prime}(F W H M)$ Gaussian, with $4 \%$ variations of the fwhm of the beam.

The brute force calculation was done by probing the beam parameter space in cosmomc in the same way as for any of the other parameters, and considering the default proposal density already implemented in cosmomc. The beam parameter is included by transforming the theoretical $C_{\ell}\left(\theta_{\mathrm{cp}}\right)$ output by CAMB at each Markov chain step into

$C_{\ell}\left(\theta_{\mathrm{cp}}, \theta_{\mathrm{b}}\right)=C_{\ell}\left(\theta_{\mathrm{cp}}\right) \times B_{\ell}^{n}+\mathcal{N}_{\ell}$,

where $f_{w h m_{n}}$ is the width of $B_{\ell}^{n}$, the Gaussian beam currently sampled. This theoretical $C_{\ell}\left(\theta_{\mathrm{cp}}, \theta_{\mathrm{b}}\right)$ is used in the Likelihood expression. To move the Markov chain to the next position in parameter space we use the default proposal density in cosmomc, usually an $N$-d Gaussian. The proposed new point is accepted/rejected following the same prescription used for the other cosmological parameters. A final marginalized distribution of the beam is output along with the other cosmological parameter constraints.

For the MCBR calculation we analysed the simulated data with a modified version of cosmomc in which the theoretical $C_{\ell}$ is multiplied by the randomly chosen beam transfer, $\mathcal{B}_{\ell}^{r}$ at each step of the chain. 

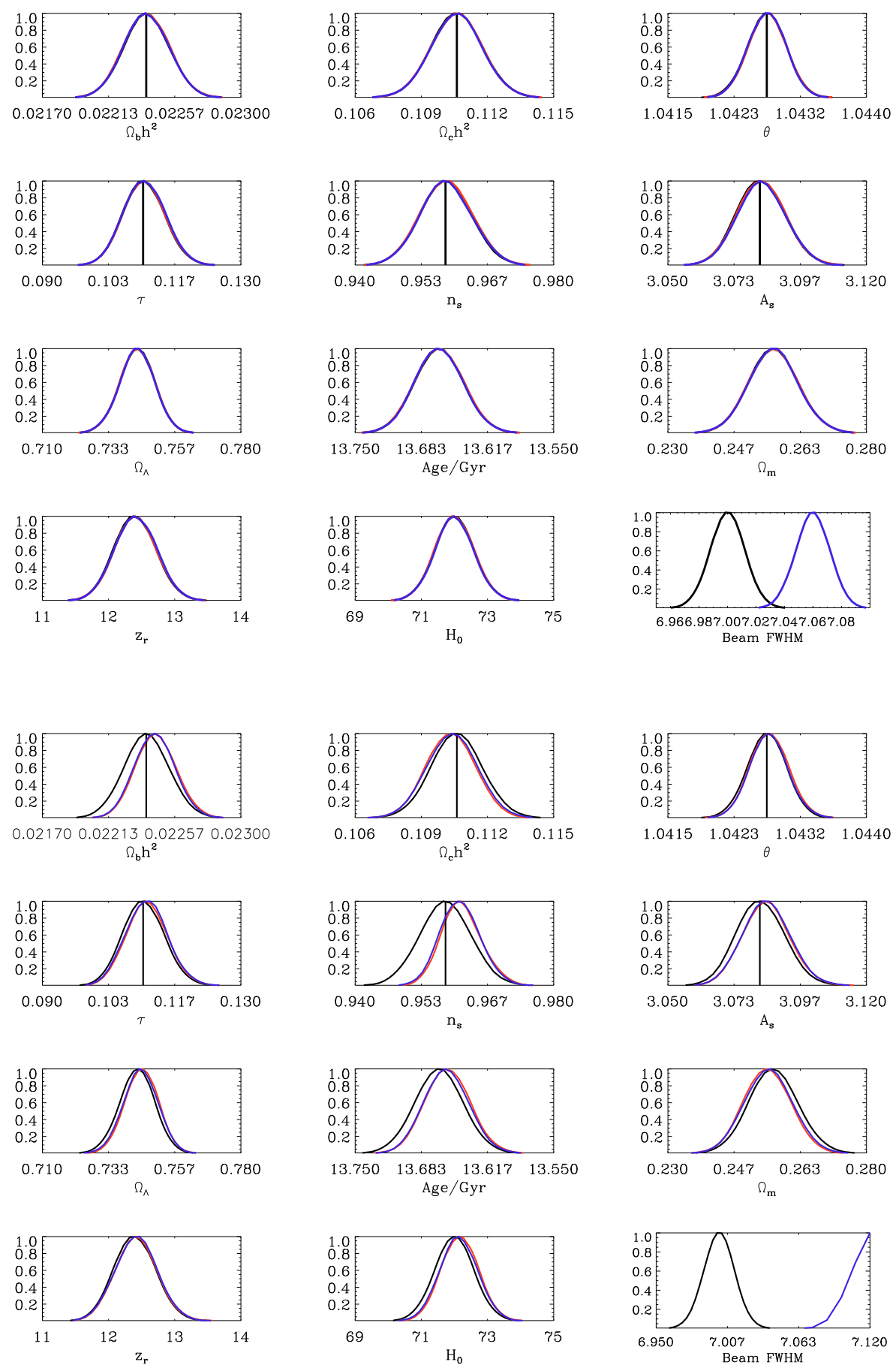
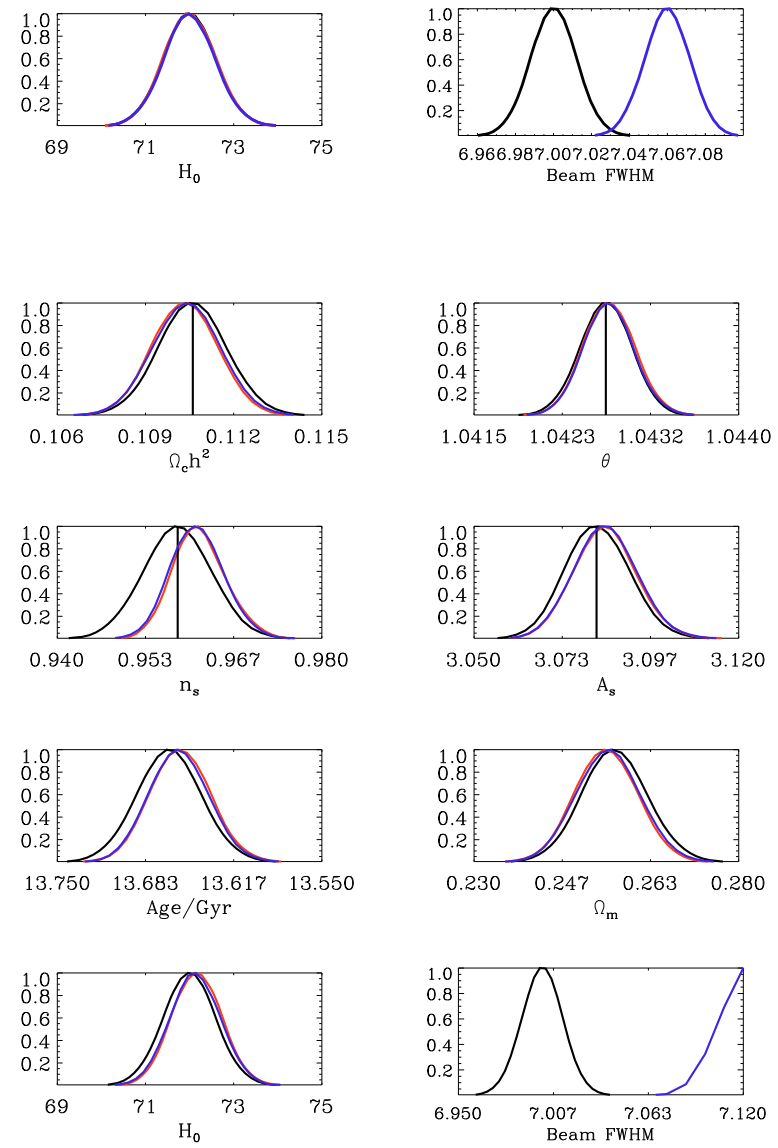
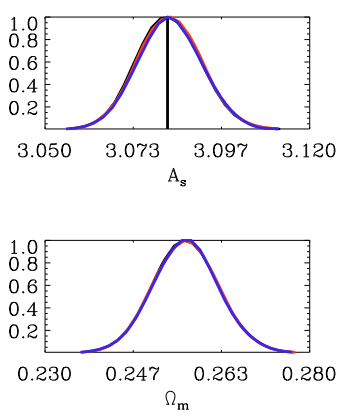

Fig. 2. Marginalized parameter constraints for Planck $143 \mathrm{GHz}$ with 7' beam with 4\% variations, for the analysis with the "true" reference fiducial beam (black) and for the mildly deviated beam transfer, $\mathcal{B}_{\ell}^{\text {mild }}=\left(B_{\ell} \times r_{\ell}^{\text {mild }}\right)^{2}$, using beamparameter approach (blue) and MCBR (red), both beamparameter and MCBR give same distributions.
Fig. 3. Marginalized parameter constraints for Planck $143 \mathrm{GHz}$ with $7^{\prime}$ beam with $4 \%$ variations, for the analysis with the "true" reference fiducial beam (black) and for the extremely deviated fiducial beam, $\mathcal{B}_{\ell}^{\text {ext }}=\left(B_{\ell} \times r_{\ell}^{\text {ext }}\right)^{2}$, using beamparameter approach (blue) and MCBR (red), both beamparameter and MCBR give same distributions.
The results are plotted in Figs. 1-3. In all cases, we find same parameter distributions for both methods. As expected, the extreme deviated beam results in a biased estimation of parameters, especially $n_{\mathrm{s}}$, but equally for both the "beamparameter" and the MCBR procedures.

\section{Beam fits and transfer function ensembles}

We characterize the beam uncertainty for Planck with a Monte Carlo ensemble of transfer functions (Huffenberger et al. 2010) generated by repeated simulation of Jupiter observations using the detector noise and pointing errors expected before flight. Each realization yields a representative transfer function.
The beams are calculated with GRASP9 (Sandri et al. 2002, 2010, in prep.; Maffei et al. 2010, in prep.; Yurchenko et al. Yurchenko et al. 2004), and we employ two methods of beam reconstruction to reproduce them from the planet scans. The first uses a rigid linearized parametric model; the second expands the beam in orthogonal functions (see Rocha et al. 2001, for a previous application of such functions in $\mathrm{CMB}$ analysis). Figure 5 shows the nominal Gaussian beams with blue-book fwhm values to that of the fiducial realistic Grasp beams based on a Gaussian fit (see Table 1). From the beam reconstruction procedure presented in Huffenberger et al. (2010) we obtain the ratio of the power spectrum as corrected with the fitted beam to the power spectrum as it should have been corrected by the true 

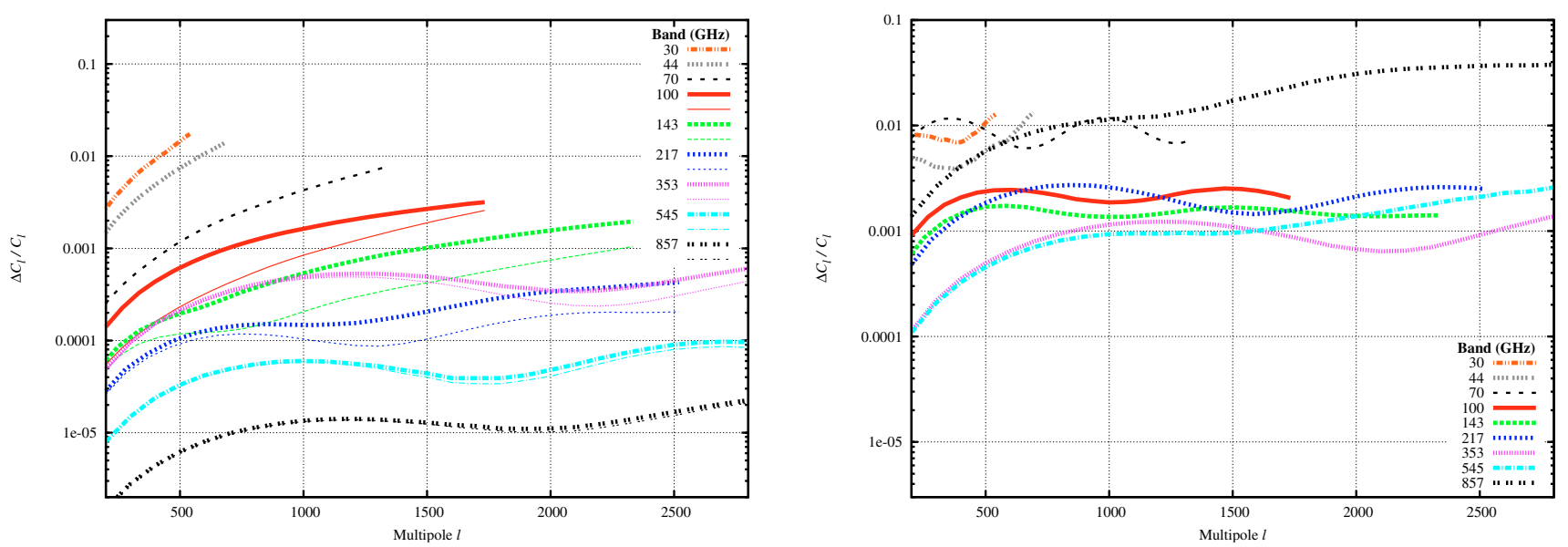

Fig. 4. At each multipole, $68 \%$ of the fitted Monte Carlo transfer functions recover spectra closer to the true power spectrum than the indicated line. Left: parametric model. Right: non-parametric model based on orthogonal functions, where the flexibility requires less knowledge of the beam, but yields larger errors.

Table 1. Planck (Planck Blue Book 2005) and Epic (Bock et al. 2008) experimental specifications ${ }^{\dagger}$.

\begin{tabular}{rcccc}
\hline \hline Experiment & Channel & $F W H M$ & $\Delta T / T$ & $F W H M$ (grasp) \\
\hline Planck & 70 & $14^{\prime}$ & 4.7 & $13^{\prime}$ \\
& 100 & $10^{\prime}$ & 2.5 & $9.22^{\prime}$ \\
& 143 & $7.1^{\prime}$ & 2.2 & $6.49^{\prime}$ \\
& 217 & $5.0^{\prime}$ & 4.8 & $4.48^{\prime}$ \\
\hline Epic-CS & 150 & $5.0^{\prime}$ & 0.81 & \\
\hline
\end{tabular}

Notes. ${ }^{(\dagger)}$ Channel frequency is given in $\mathrm{GHz}, F W H M$ in arcminutes and noise in $10^{-6}$. The last column gives the fwhm of our fiducial beams based on a Gaussian fit to the realistic GRASP beams.

beam. In Fig. 4 we display lines which bound $68 \%$ of the ensemble transfer functions for Planck channels.

The simulation of repeated Jupiter calibrations is done in such way that each template is an unbiased estimator of the true template. But in real life, they could be a biased estimator (for instance the Planck pointing error could bias the beam function always in the same direction). This prompted us to consider the runs presented in Sect. 5.2.

The Beam fitting is applied to data with white $+1 / f$ noise, and to destriped data, i.e., after application e.g. of a "destriping" mapmaking code which removes almost all of the effects of $1 / f$ noise (Poutanen et al. 2006; Ashdown et al. 2007a,b, 2009). We use realistic Grasp beams and the parametric model of the reconstructed beams (the results with non-parametric model will be presented in a future paper). Figure 6 shows extreme and mild beam transfer functions for the Planck $70 \mathrm{GHz}$, $100 \mathrm{GHz}, 143 \mathrm{GHz}$ and $217 \mathrm{GHz}$ channels obtained from the beam fitting procedure applied to destriped data (hence containing a a very low level of $1 / f$ residuals). For comparison purposes we plot in Fig. 7 these functions obtained from data with a white and $1 / f$ noise background. We also plot in Figs. 8 the normalized histograms of the ratios, $r_{\ell}^{2}$, for singe multipoles $\ell=500,1000,1500,2000$. For all channels the distributions are slightly skewed and get broader with increasing multipole $\ell$ for each channel. We can also compare the probability of the mildly deviated and extremely deviated transfer functions used in Sect. 5. For instance for $70 \mathrm{GHz}$ for $\ell=1000$

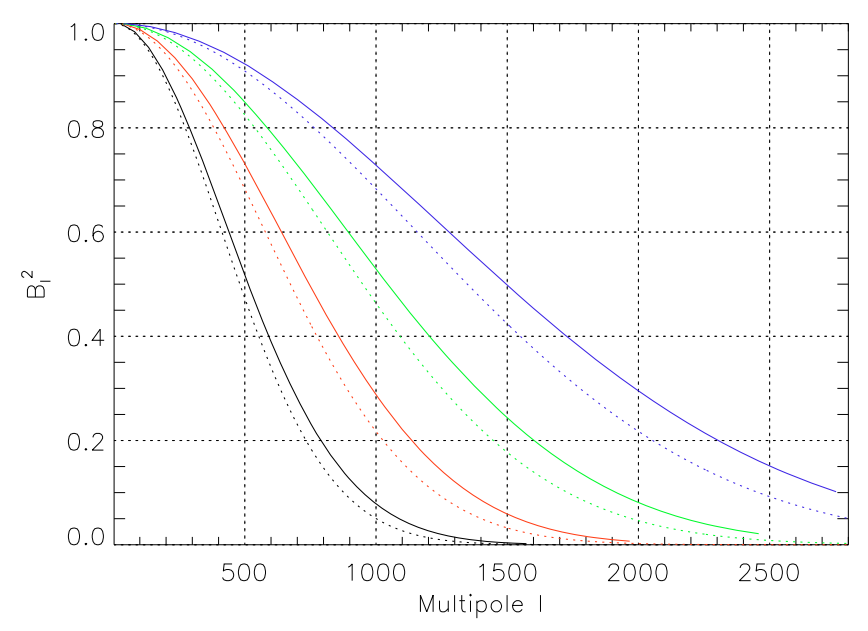

Fig. 5. Nominal Gaussian blue-book beams (dotted line) vs. Fiducial realistic Grasp9 beams based on a Gaussian fit (solid line) for $70 \mathrm{GHz}$ (black), $100 \mathrm{GHz}$ (red), $143 \mathrm{GHz}$ (green) and $217 \mathrm{GHz}$ (blue).

the mild function is $\simeq 10 \%$ probable while the extreme function is approximately 100 times less likely. The maximum variation for transfer function ratios, $r_{\ell}^{2}$, is of the order $2 \%$ for $70 \mathrm{GHz}$ $(0.5 \%$ for $100 \mathrm{GHz})$ for destriped data, while for white and $1 / f$ noise data with no attempt at destriping it increases to $\sim 30 \%$ for the $70 \mathrm{GHz}$ channel ( $2.5 \%$ for $100 \mathrm{GHz})$.

In Sect. 5 we infer that the parameter constraints from beams obtained with destriped data are slightly worse but very close to those obtained with a white noise background as expected.

\section{Analysis: from Beam transfer function uncertainties to parameter estimation}

To propagate the beam measurement errors to parameters we apply the MCBR method following the procedure described in Sect. 2. We make use of the beam transfer functions obtained with the beam fitting described in Sect. 3. For this purpose we use a modified version of cosmomc with built-in MCBR step as described in Sect. 2. We consider a set of five chains. The convergence diagnostic is based on the Gelman and Rubin statistic, 

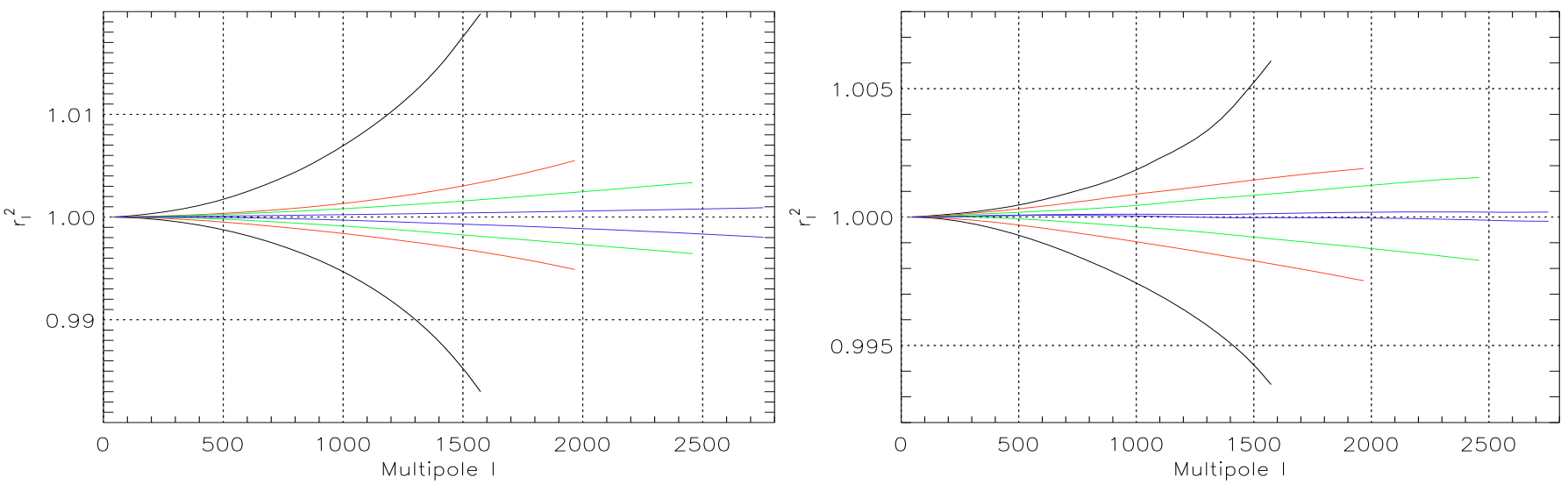

Fig. 6. Extreme (left) and Mild (right) beam transfer functions for the Planck $70 \mathrm{GHz}$ (black), $100 \mathrm{GHz}$ (red), $143 \mathrm{GHz}$ (green) and $217 \mathrm{GHz}$ (blue) channels obtained from beam fitting applied to destriped data.
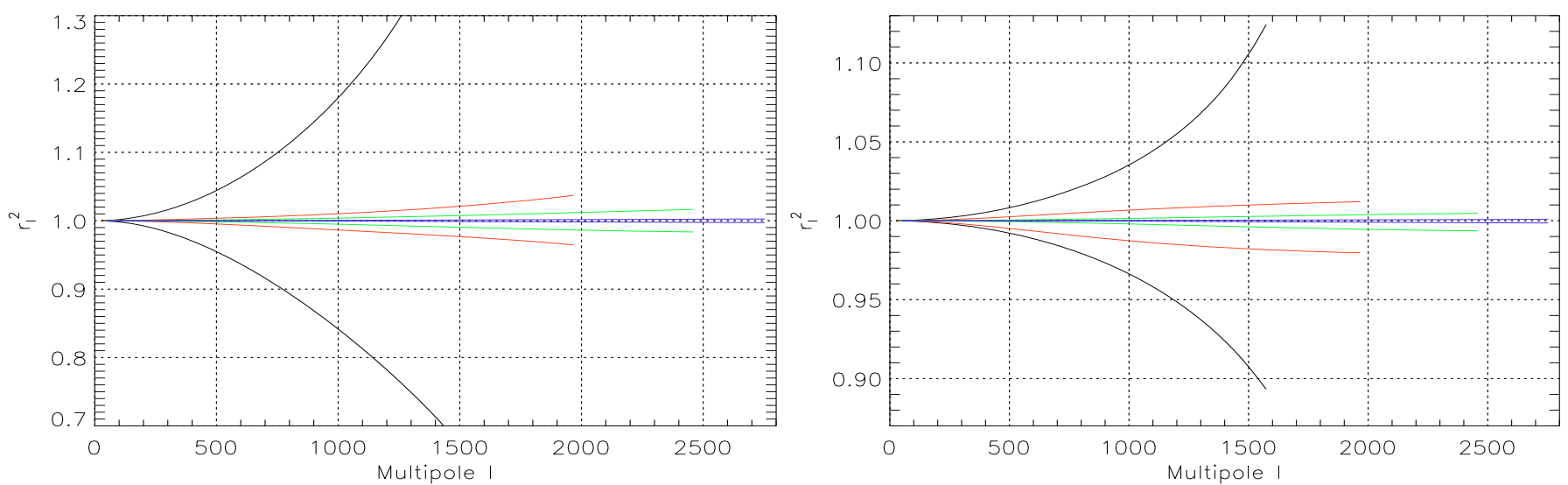

Fig. 7. Extreme (left) and Mild (right) beam transfer functions for the Planck $70 \mathrm{GHz}$ (black), $100 \mathrm{GHz}$ (red), $143 \mathrm{GHz}$ (green) and $217 \mathrm{GHz}$ (blue) channels obtained from beam fitting applied to data with white $+1 / f$ noise.
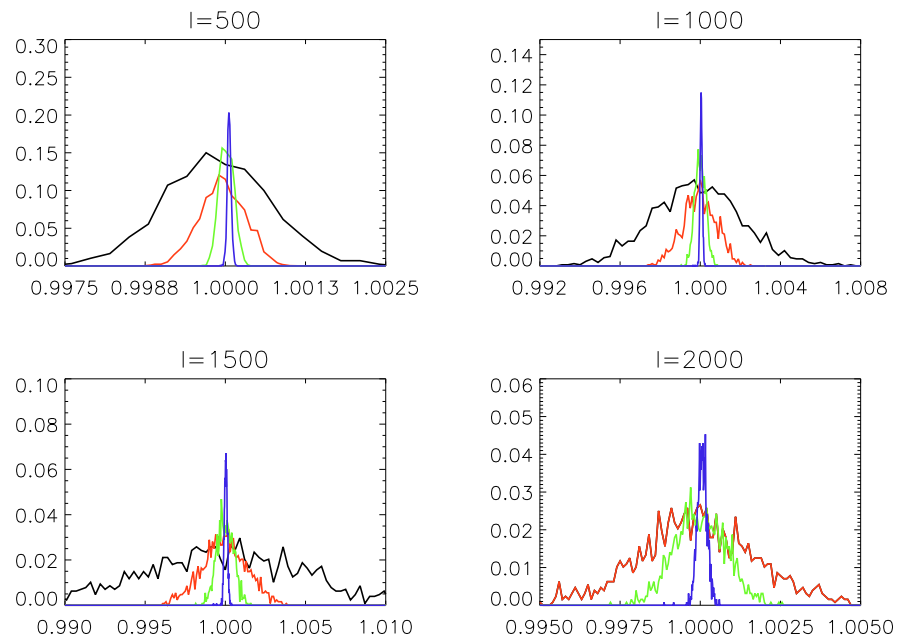

Fig. 8. Normalized distributions of the beam transfer functions, $\mathcal{B}_{\ell}=$ $\left(B_{\ell}^{r}\right)^{2}$ for multipoles $\ell=500,1000,1500,2000$ for $70 \mathrm{GHz}$ (black), $100 \mathrm{GHz}$ (red), $143 \mathrm{GHz}$ (green), and $217 \mathrm{GHz}$ (blue) obtained from the beam fitting on destriped data.

as usual in the field. Following MCBR, we choose randomly the beam transfer function (from the set of 1280 simulations) for

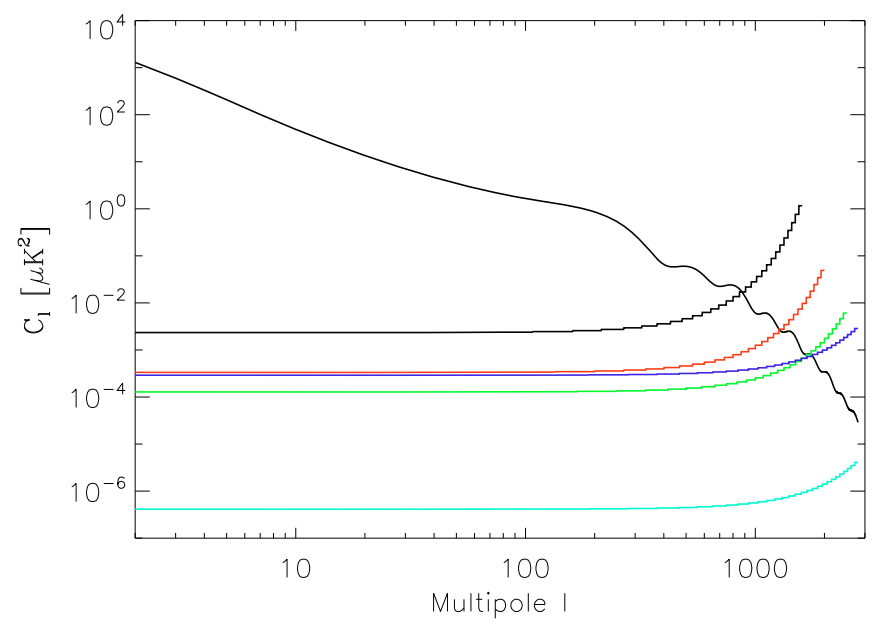

Fig. 9. CMB angular power spectrum (best fit of WMAP 1 yr, black line) and noise levels for Planck: $70 \mathrm{GHz}$ (black), $100 \mathrm{GHz}$ (red), $143 \mathrm{GHz}$ (green), $217 \mathrm{GHz}$ (blue) and for Epic $150 \mathrm{GHz}$ (cian).

each step of the markov chain. We sample a six-dimensional set of cosmological parameters, with flat priors: the physical baryon 
G. Rocha et al.: MCBR: a study of the impact of PLANCK beam measurement errors on cosmological parameter estimation
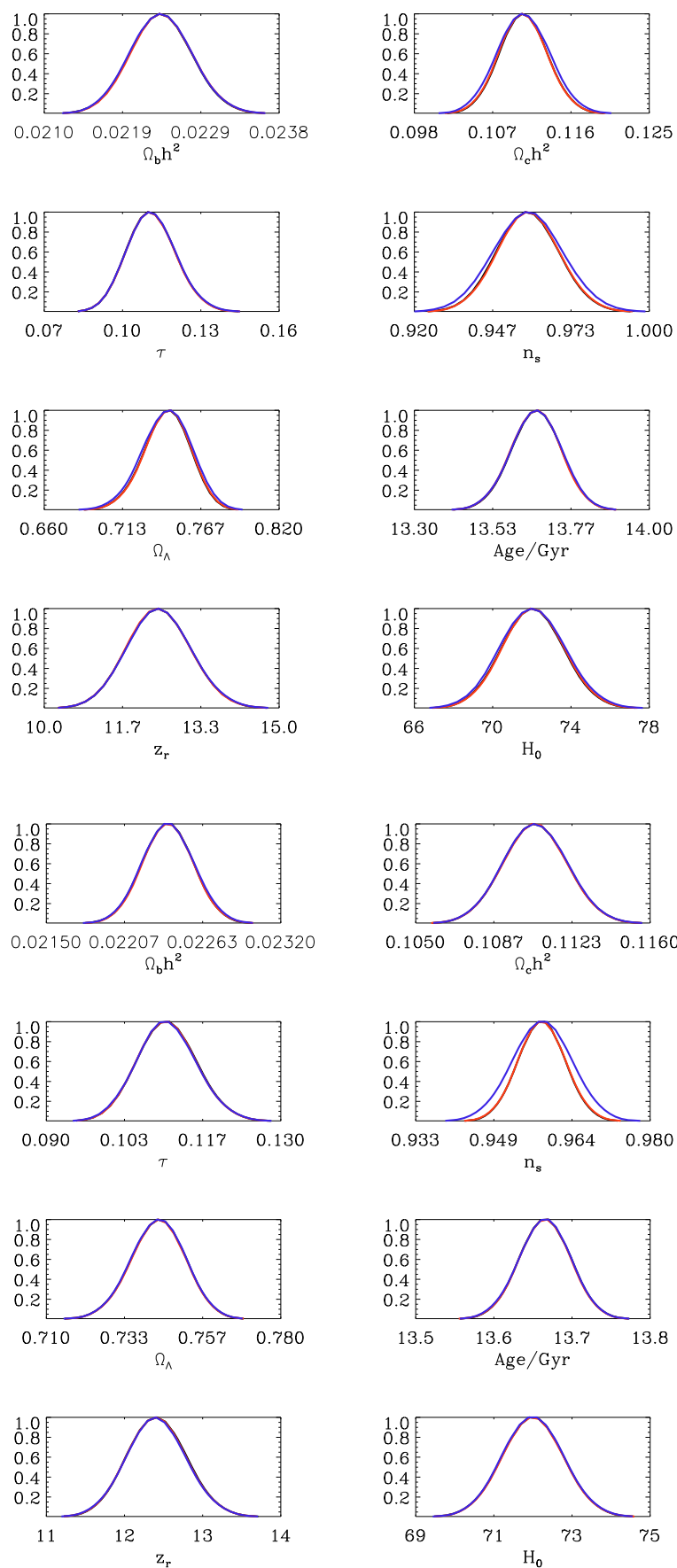
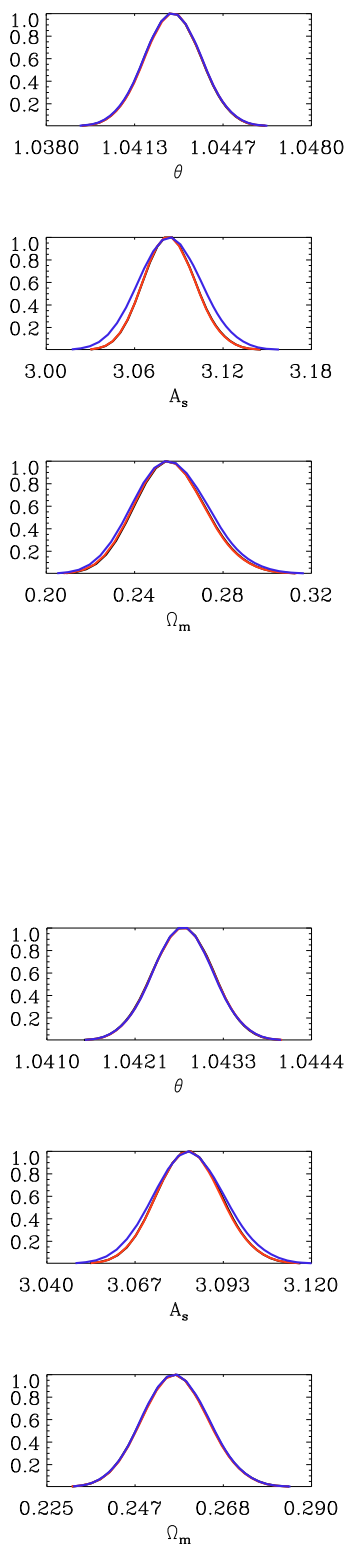

Fig. 10. Marginalized parameter constraints for Planck $70 \mathrm{GHz}$ without beam uncertainty (black), marginalized over the beam uncertainty via MCBR considering the destriped data (red), and in the presence of white noise $+1 / f$ noise (blue).

Fig. 11. Marginalized parameter constraints for Planck $100 \mathrm{GHz}$ without beam uncertainty (black), marginalized over the beam uncertainty via MCBR considering the destriped data (red) and in the presence of white noise $+1 / f$ noise (blue). and Cold Dark Matter densities, $\omega_{\mathrm{b}}=\Omega_{\mathrm{b}} h^{2}$ and $\omega_{\mathrm{c}}=\Omega_{\mathrm{c}} h^{2}$; the ratio of the sound horizon to the angular diameter distance at decoupling, $\theta_{\mathrm{S}}$; the scalar spectral index $n_{\mathrm{S}}$; the overall normalization of the spectrum $\log \left[10^{10} A\right]$ at $k=0.05 \mathrm{Mpc}^{-1}$ (hereafter $A_{\mathrm{S}}$ ), and the optical depth to reionization $\tau$. We use a cosmic age top-hat prior $10 \mathrm{Gyr} \leq t_{0} \leq 20 \mathrm{Gyr}$, consider purely adiabatic initial conditions only, we impose flatness, and we treat the dark energy component as a cosmological constant.

We create simulated datasets with the noise properties of the Planck 70, 100, 143 and $217 \mathrm{GHz}$ (Planck Blue Book 2005) channels, as well as one example of a future experiment. For the latter we considered the noise levels of Epic $150 \mathrm{GHz}$ (Bock et al. 2008). We take as our cosmological model the best fit of WMAP 1 yr: $\Omega_{\mathrm{b}} h^{2}=0.02238 ; \Omega_{\mathrm{c}} h^{2}=0.11061 ; H_{0}=71.992$; $\tau=0.110267 ; n_{\mathrm{S}}=0.95820 ;$ and $A_{\mathrm{S}}=3.0824$ (Spergel et al. 2003). These simulated datasets are given in terms of the angular Power Spectrum $C_{\ell}^{\mathrm{obs}}$ as described in Sect. 2. We compute the noise $\mathcal{N}_{\ell}=(\Delta T \times f w h m)^{2}$ for Planck and Epic from the sensitivity $\Delta T / T$ and the nominal fwhm of the beam assuming a Gaussian profile (tabulated in Table 1). In Fig. 9 we plot the theoretical model vs. the noise levels for each channel considered. Results from this analysis are given in Sect. 5.

\section{Results}

\subsection{Results: effect of beam uncertainties}

Figures 10-13 show the marginalized parameter constraints for Planck in three cases: without beam uncertainty (i.e., 

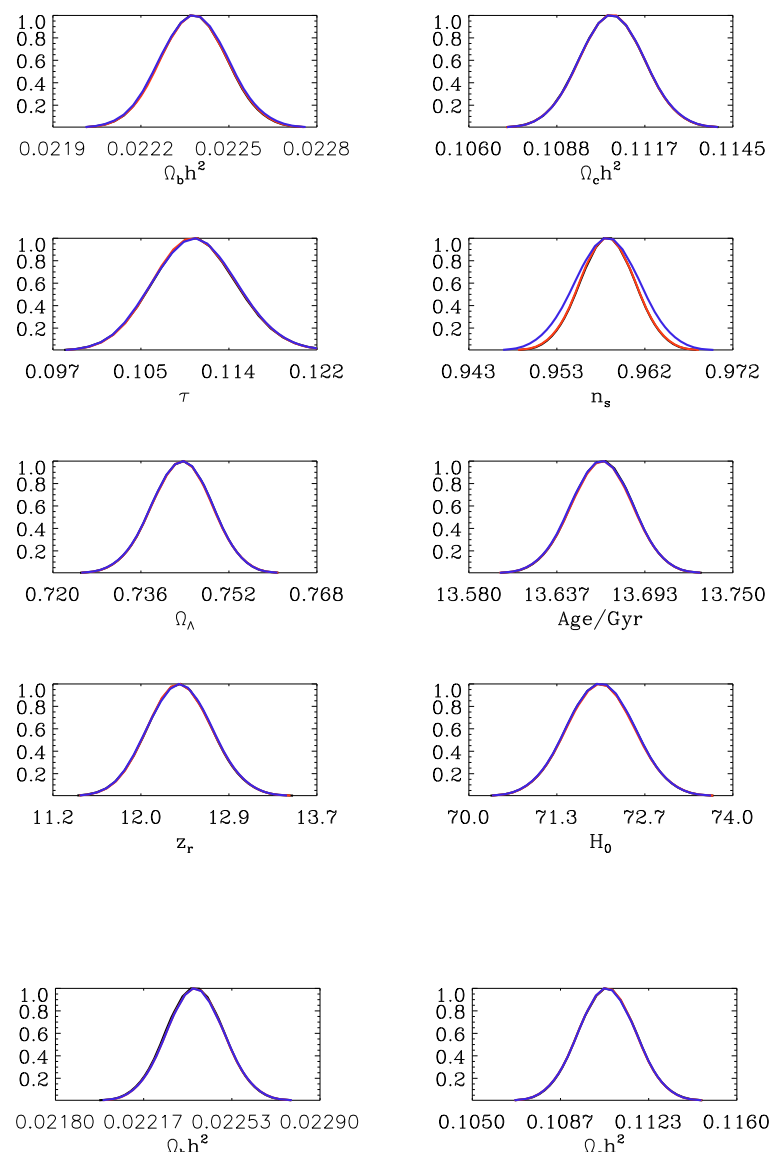

$\Omega_{\mathrm{b}} \mathrm{h}$
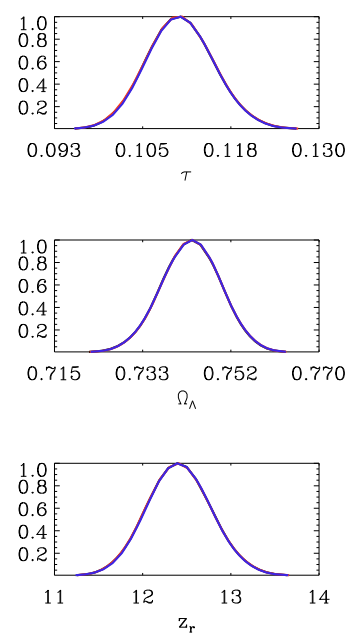
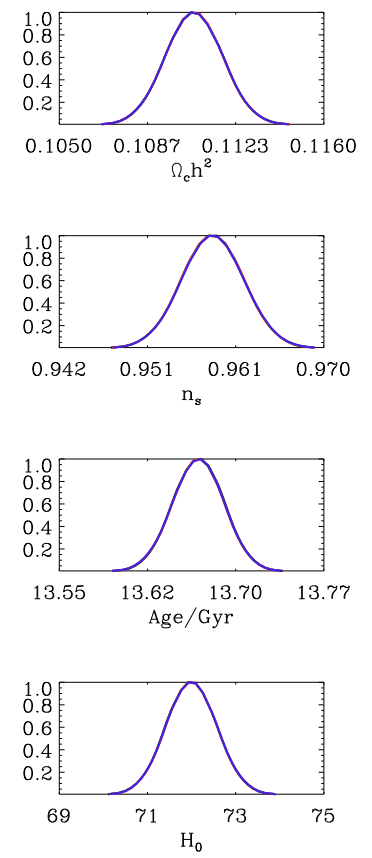
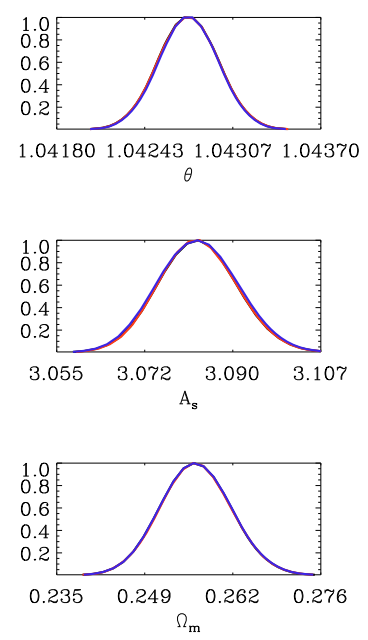

Fig. 12. Marginalized parameter constraints for Planck $143 \mathrm{GHz}$ without beam uncertainty (black), marginalized over the beam uncertainty MCBR considering the destriped data (red) and in the presence of white noise $+1 / f$ noise (blue).
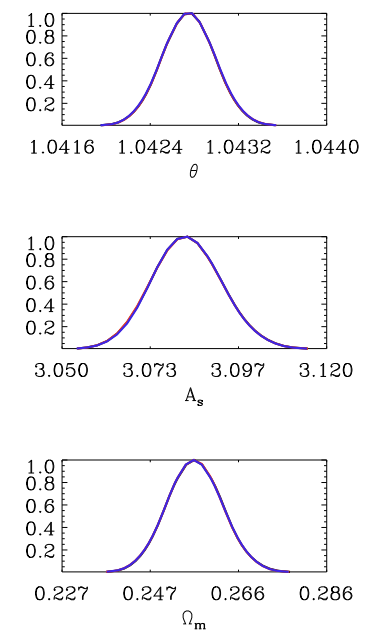

considering the true fiducial beam) (black); with beam uncertainty using the beam transfer functions obtained using the destriped data (red); and in the presence of $1 / f$ noise (blue). Table 2 gives the input cosmological parameters and the mean values and marginalized $68 \%$ confidence limits obtained after accounting for the beam errors. To facilitate comparisons, Fig. 14 shows these same marginalized constraints for $n_{\mathrm{s}}$ and $A_{\mathrm{s}}$, the parameters where the largest differences are seen between the three cases.

Equivalent results for a more sensitive polarization experiment - Epic $150 \mathrm{GHz}$ - are plotted in Fig. 15; corresponding parameter values are given in Table 2.
The most noticeable effect of beam uncertainties is to widen the marginal distributions of some parameters, especially $n_{\mathrm{s}}$, for uncertainties obtained in the presence of $1 / f$ noise but without destriping. $A_{\mathrm{s}}$, and $\Omega_{\mathrm{b}} h^{2}$ are also affected. In this case the distribution of the fitted beam transfer functions is wider than that obtained from white noise or destriped data as shown in Figs. 6 and 7.

Define $\sigma_{\text {ch }}$, the width of the distribution when beam errors are marginalised by applying MCBR, and $\sigma_{\text {ref }}$, the width of the distribution for the simulated data convolved with the fiducial beam (with no beam errors included). Figure 16 shows the enhancement factor, $\sigma_{\mathrm{ch}} / \sigma_{\text {ref }}$, for parameters $n_{\mathrm{s}}$ and $A_{\mathrm{s}}$ 
G. Rocha et al.: MCBR: a study of the impact of PLANCK beam measurement errors on cosmological parameter estimation

Table 2. Mean values and marginalized $68 \%$ c.l. limits using the fiducial beam: analysis without beam uncertainty (Col. 3), accounting the beam uncertainty from destriped data (Col. 4) and from the data with white and $1 / f$ noise (Col. 5).

\begin{tabular}{|c|c|c|c|c|}
\hline Channel & Parameter & No beam uncertainty & Destriped & White noise $+1 / f$ \\
\hline \multirow[t]{6}{*}{ Planck $70 \mathrm{GHz}$} & $\Omega_{\mathrm{b}} h^{2}$ & $0.22393 \pm 0.00035$ & $0.22401 \pm 0.00035$ & $0.22394 \pm 0.00036$ \\
\hline & $\Omega_{\mathrm{c}} h^{2}$ & $0.1106 \pm 0.0027$ & $0.1105 \pm 0.0027$ & $0.1106 \pm 0.0029$ \\
\hline & $\theta$ & $1.0428 \pm 0.0010$ & $1.0428 \pm 0.0010$ & $1.0428 \pm 0.0010$ \\
\hline & $\tau$ & $0.1112 \pm 0.0091$ & $0.1111 \pm 0.0091$ & $0.1112 \pm 0.0091$ \\
\hline & $n_{\mathrm{s}}$ & $0.959 \pm 0.010$ & $0.959 \pm 0.010$ & $0.959 \pm 0.011$ \\
\hline & $A_{\mathrm{s}}$ & $3.084 \pm 0.017$ & $3.084 \pm 0.017$ & $3.084 \pm 0.021$ \\
\hline \multirow[t]{6}{*}{ Planck $100 \mathrm{GHz}$} & $\Omega_{\mathrm{b}} h^{2}$ & $0.22383 \pm 0.00018$ & $0.22383 \pm 0.00018$ & $0.22383 \pm 0.00018$ \\
\hline & $\Omega_{\mathrm{c}} h^{2}$ & $0.1106 \pm 0.0015$ & $0.1106 \pm 0.0015$ & $0.1106 \pm 0.0015$ \\
\hline & $\theta$ & $1.04275 \pm 0.00038$ & $1.04275 \pm 0.00038$ & $1.04275 \pm 0.00038$ \\
\hline & $\tau$ & $0.1107 \pm 0.0049$ & $0.1106 \pm 0.0049$ & $0.1106 \pm 0.0049$ \\
\hline & $n_{\mathrm{s}}$ & $0.9583 \pm 0.0046$ & $0.9583 \pm 0.0046$ & $0.9583 \pm 0.0058$ \\
\hline & $A_{\mathrm{s}}$ & $3.0832 \pm 0.0094$ & $3.0831 \pm 0.0094$ & $3.083 \pm 0.011$ \\
\hline \multirow[t]{6}{*}{ Planck $143 \mathrm{GHz}$} & $\Omega_{\mathrm{b}} h^{2}$ & $0.22381 \pm 0.00011$ & $0.22381 \pm 0.00011$ & $0.22381 \pm 0.00011$ \\
\hline & $\Omega_{\mathrm{c}} h^{2}$ & $0.1106 \pm 0.0010$ & $0.1106 \pm 0.0010$ & $0.1106 \pm 0.0010$ \\
\hline & $\theta$ & $1.04275 \pm 0.00021$ & $1.04275 \pm 0.00021$ & $1.04274 \pm 0.00021$ \\
\hline & $\tau$ & $0.1105 \pm 0.0038$ & $0.1105 \pm 0.0038$ & $0.1105 \pm 0.0039$ \\
\hline & $n_{\mathrm{s}}$ & $0.9582 \pm 0.0029$ & $0.9582 \pm 0.0029$ & $0.9582 \pm 0.0035$ \\
\hline & $A_{\mathrm{s}}$ & $3.0829 \pm 0.0074$ & $3.0828 \pm 0.0075$ & $3.0829 \pm 0.0077$ \\
\hline \multirow[t]{6}{*}{ Planck $217 \mathrm{GHz}$} & $\Omega_{\mathrm{b}} h^{2}$ & $0.22380 \pm 0.00012$ & $0.22382 \pm 0.00012$ & $0.22383 \pm 0.00012$ \\
\hline & $\Omega_{\mathrm{c}} h^{2}$ & $0.1106 \pm 0.0012$ & $0.1106 \pm 0.0012$ & $0.1106 \pm 0.0012$ \\
\hline & $\theta$ & $1.04275 \pm 0.00023$ & $1.04275 \pm 0.00023$ & $1.04275 \pm 0.00024$ \\
\hline & $\tau$ & $0.1106 \pm 0.0046$ & $0.1106 \pm 0.0046$ & $0.1107 \pm 0.0047$ \\
\hline & $n_{\mathrm{s}}$ & $0.9582 \pm 0.0032$ & $0.9583 \pm 0.0032$ & $0.9583 \pm 0.0032$ \\
\hline & $A_{\mathrm{s}}$ & $3.0831 \pm 0.0090$ & $3.0830 \pm 0.0090$ & $3.0831 \pm 0.0090$ \\
\hline \multirow[t]{6}{*}{ Epic $150 \mathrm{GHz}$} & $\Omega_{\mathrm{b}} h^{2}$ & $0.223802 \pm 0.000029$ & $0.223798 \pm 0.000029$ & $0.223802 \pm 0.000029$ \\
\hline & $\Omega_{\mathrm{c}} h^{2}$ & $0.11061 \pm 0.00051$ & $0.11061 \pm 0.00051$ & $0.11061 \pm 0.00052$ \\
\hline & $\theta$ & $1.042750 \pm 0.000054$ & $1.042750 \pm 0.000054$ & $1.042750 \pm 0.00054$ \\
\hline & $\tau$ & $0.1104 \pm 0.0023$ & $0.1103 \pm 0.0023$ & $0.1103 \pm 0.0024$ \\
\hline & $n_{\mathrm{s}}$ & $0.9582 \pm 0.0016$ & $0.9582 \pm 0.0016$ & $0.9583 \pm 0.0016$ \\
\hline & $A_{\mathrm{s}}$ & $3.0827 \pm 0.0047$ & $3.0825 \pm 0.0047$ & $3.083 \pm 0.0047$ \\
\hline
\end{tabular}
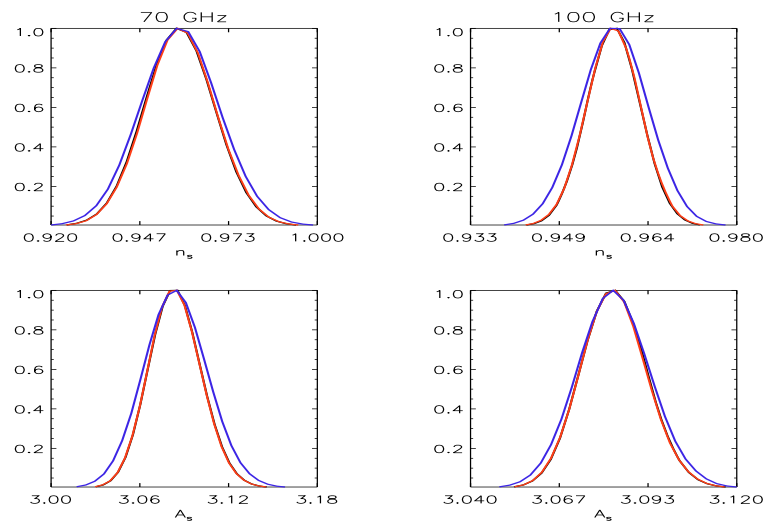
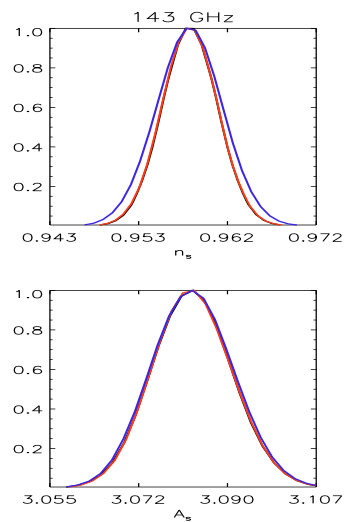

Fig. 14. Marginalized constraints for the most impacted parameters, $n_{\mathrm{s}}$ and $A_{\mathrm{s}}$, for Planck channels $70 \mathrm{GHz}, 100 \mathrm{GHz}$ and $143 \mathrm{GHz}$, without beam uncertainty (black), marginalized over the beam uncertainty considering the destriped data (red) and in the presence of white noise $+1 / f$ noise (blue). for beams fitted on data with white $+1 / f$ noise. For example, at $100 \mathrm{GHz}$ the distributions of $n_{\mathrm{s}}$ and $A_{\mathrm{s}}$ widen by $25 \%$ and $11 \%$, respectively.
This widening is much reduced by the use of destriping techniques. For example, with destriping the uncertainties in the beams are $0.5 \%$ for $100 \mathrm{GHz}$ at $\ell=1500$, which translates into 

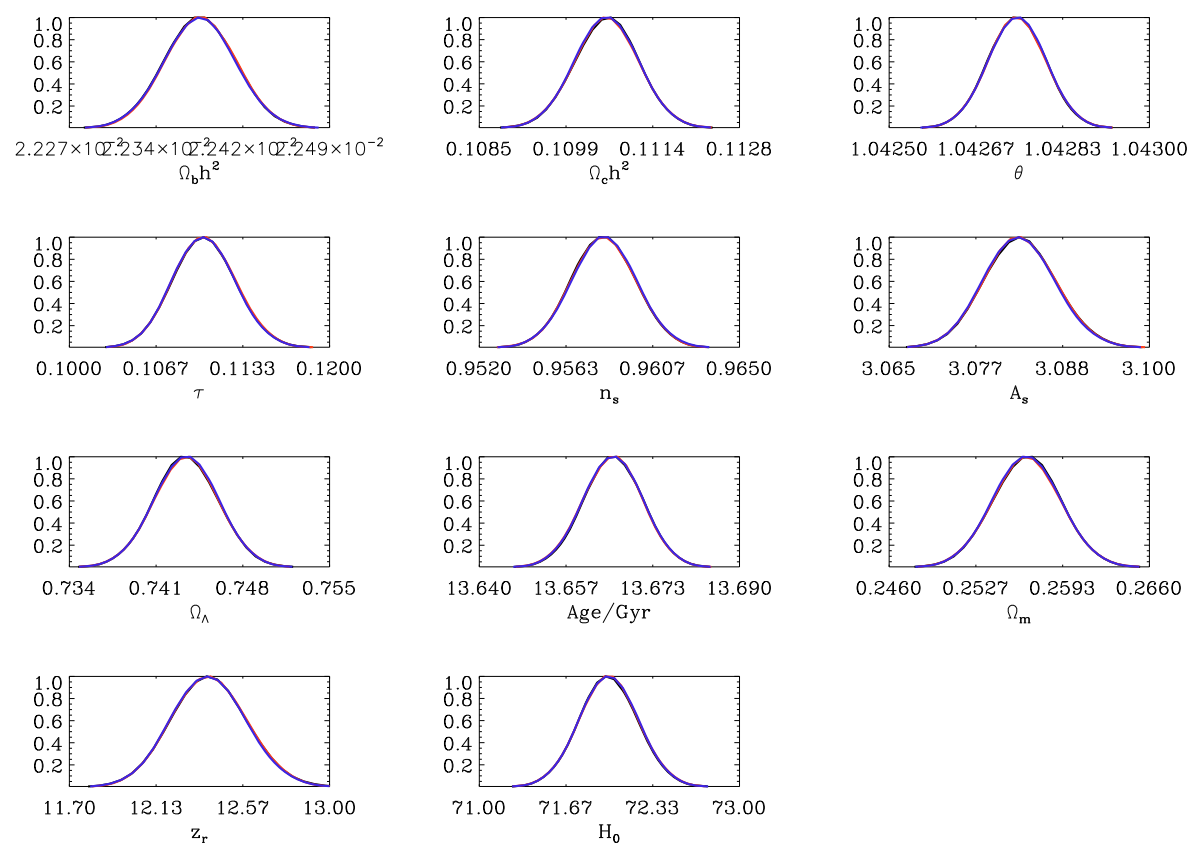

Fig. 15. Marginalized parameter constraints for a future experiment with Epic $150 \mathrm{GHz}$ specifications without beam uncertainty (black), marginalized over the beam uncertainty considering the destriped data (red) and in the presence of white noise $+1 / f$ noise (blue).
Table 3. Bias on $n_{\mathrm{s}}$ and $A_{\mathrm{s}}$ in units of the error due to the deviation of the extreme function $\left(r_{\ell}^{\text {ext }}\right)^{2}$ at $\ell=1 / \sigma$, after MCBR, fitted on destriped data. For each Planck channel.

\begin{tabular}{lcccc}
\hline \hline ch & $\ell$ & $r_{\ell}^{2}$ & bias $/ \sigma\left(n_{\mathrm{s}}\right)$ & bias $/ \sigma\left(A_{\mathrm{s}}\right)$ \\
\hline 70 & 579 & 1.00217 & 0.1890 & 0.2846 \\
100 & 810 & 1.000928 & 0.3282 & 0.1240 \\
143 & 1141 & 1.000982 & 0.3775 & 0.1055 \\
217 & 1620 & 1.00043 & 0.0929 & 0.0188 \\
\hline
\end{tabular}

an increase of parameter uncertainties of $0.1 \%$. Without destriping, the uncertainties on $A_{\mathrm{s}}$ at $70 \mathrm{GHz}$ and on $n_{\mathrm{s}}$ at $100 \mathrm{GHz}$ increase by $21 \%$ and $25 \%$, respectively, for beams fitted on white + $1 / f$ noise data. This is a convincing demonstration of the relevance and power of destriping techniques in reducing the effect of $1 / f$ noise for Planck.

\subsection{Results: effect of assuming a wrong fiducial beam}

To illustrate the effect of incorrect beam assumptions we calculated parameters assuming a mildly and then an extremely "wrong" beam. Specifically, we generated three simulated datasets, using: $\mathcal{B}_{\ell}$; a mildly wrong beam $\mathcal{B}_{\ell}^{\text {mild }}$; and an extremely wrong beam $\mathcal{B}_{\ell}^{\text {ext }}$. We analyzed these datasets with the modified version of cosmomc with MCBR built in, and compared the cosmological parameters from the run with for the "true" fiducial beam $\mathcal{B}_{\ell}$ to those of both the mild and extreme deviated beams.

Figures 17-20 show marginalized parameter constraints from $70 \mathrm{GHz}, 100 \mathrm{GHz}, 143 \mathrm{GHz}$, and $217 \mathrm{GHz}$, respectively, on destriped data. We see that assuming an extreme beam deviation in the simulated data results in a biased estimation of some parameters, particularly $n_{\mathrm{s}}$. This is mostly due to incomplete

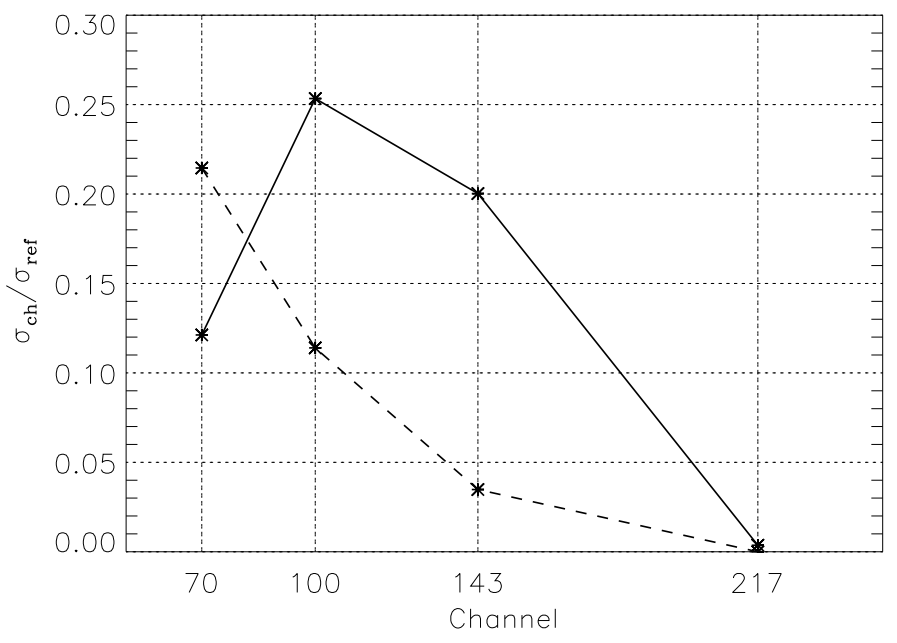

Fig. 16. Enhancement factor, $\sigma_{\mathrm{ch}} / \sigma_{\text {ref }}$ for $n_{\mathrm{s}}$ (solid line) and $A_{\mathrm{s}}$ (dashed line), where $\sigma_{\mathrm{ch}}$ is the width of the distribution when beam errors are marginalized over by applying MCBR; $\sigma_{\text {ref }}$ is the width of the distribution for the simulated data convolved with the fiducial beam (no beams errors included), for beams fitted on data with white and $1 / f$ noise background.

marginalization, as we do not encompass an adequate distribution of deviations from the chosen fitted transfer function.

For comparison, Figs. 21 and 22 show marginalized parameter constraints for the $100 \mathrm{GHz}$ and $143 \mathrm{GHz}$ channels, respectively, on data that have not been destriped.

Figure 23 shows the bias in $n_{\mathrm{s}}$ and $A_{\mathrm{s}}$ as a function of the extreme beams fitted on destriped data. We consider the error on $B_{\ell}$ given by $\left(r_{\ell}-1\right)$ for $\ell=1 / \sigma$ representing the sigma of the beam. The corresponding values are given in Table 3 . For example for $100 \mathrm{GHz}$ an uncertainty of the extreme beam transfer function $b_{\ell}^{2}$ for $\ell=810$ of $\simeq 0.1 \%$ bias the likelihood by $0.3 \sigma$ and $0.13 \sigma$ for $n_{\mathrm{s}}$ and $A_{\mathrm{s}}$ respectively. A beam transfer function 

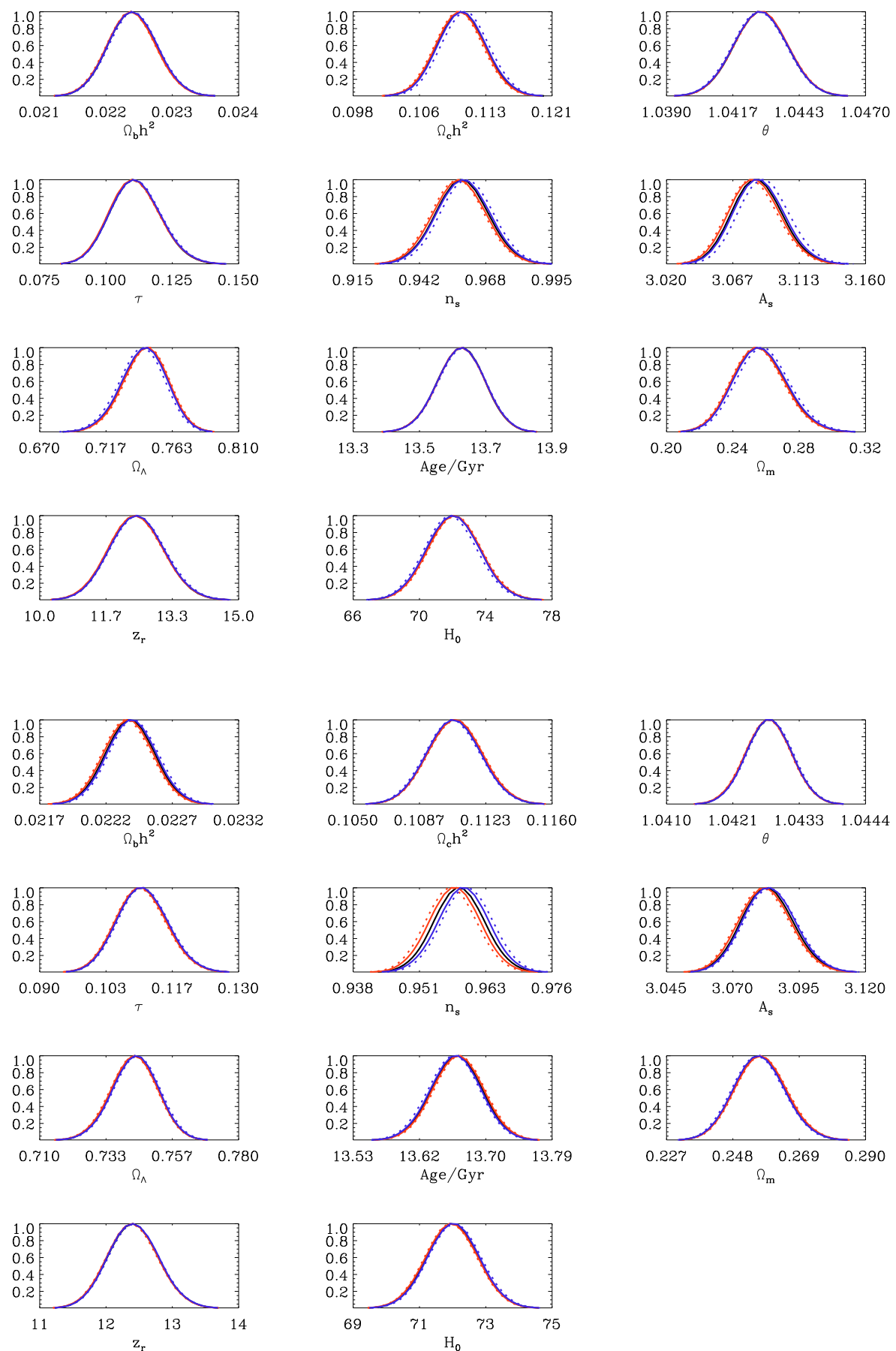

Fig. 17. Marginalized parameter constraints for Planck $70 \mathrm{GHz}$ with beam randomization MCBR: true beam (black), decreasing function for destriped data (red), increasing function for destriped data (blue), mild deviation (solid line) and extreme deviation from the true beam (dotted line).

Fig. 18. Marginalized parameter constraints for Planck $100 \mathrm{GHz}$ with beam randomization MCBR: true beam (black), decreasing function for destriped data (red), increasing function for destriped data (blue), mild deviation (solid line) and extreme deviation from the true beam (dotted line). known up to $0.02 \%$ will bias $n_{\mathrm{s}}$ by $0.1 \sigma$. If we had not taken into account the beam uncertainties, then the same deviation in the transfer functions would have biased $n_{\mathrm{s}}$ by as much as $0.4 \sigma$, as can be inferred from Fig. 16. The inadequacy of a likelihood that does not integrate the beam uncertainties is mentioned in (Huffenberger et al. 2010). There a simplified analysis of noisier data (only 1 horn) with all parameters except $n_{\mathrm{s}}$ fixed indicated that limiting the bias to $0.1 \sigma$ would require knowledge of $b_{\ell}^{2}$ to $0.04 \%$ where it has fallen to $1 \%$ of peak $(\ell \simeq 1900$ for $100 \mathrm{GHz}$ ). In our analysis here we see that at $\ell \simeq 1900$ an uncertainty of $0.5 \%$ for the extreme function would bias $n_{\mathrm{s}}$ by $0.3 \sigma$, while a mild deviation of the order $0.2 \%$ would produce a bias below $0.05 \sigma$ (see Table 2). Hence a beam deviation five times that reported in Huffenberger et al. (2010) would bias $n_{\mathrm{s}}$ by less than $0.1 \sigma$. This improvement is mostly due to properly marginalizing over the beam uncertainties via the MCBR method.

\section{Conclusions}

We have developed a fast new method, MCBR, to propagate beam uncertainties to parameter estimation. The method properly accounts for the marginalised errors in the parameters. A desirable feature of the method is that it makes minimal assumptions on beam uncertainties. For example, it does not assume the data are normally distributed, and, unlike other approaches such as analytic marginalization, it does not require Gaussian priors on 

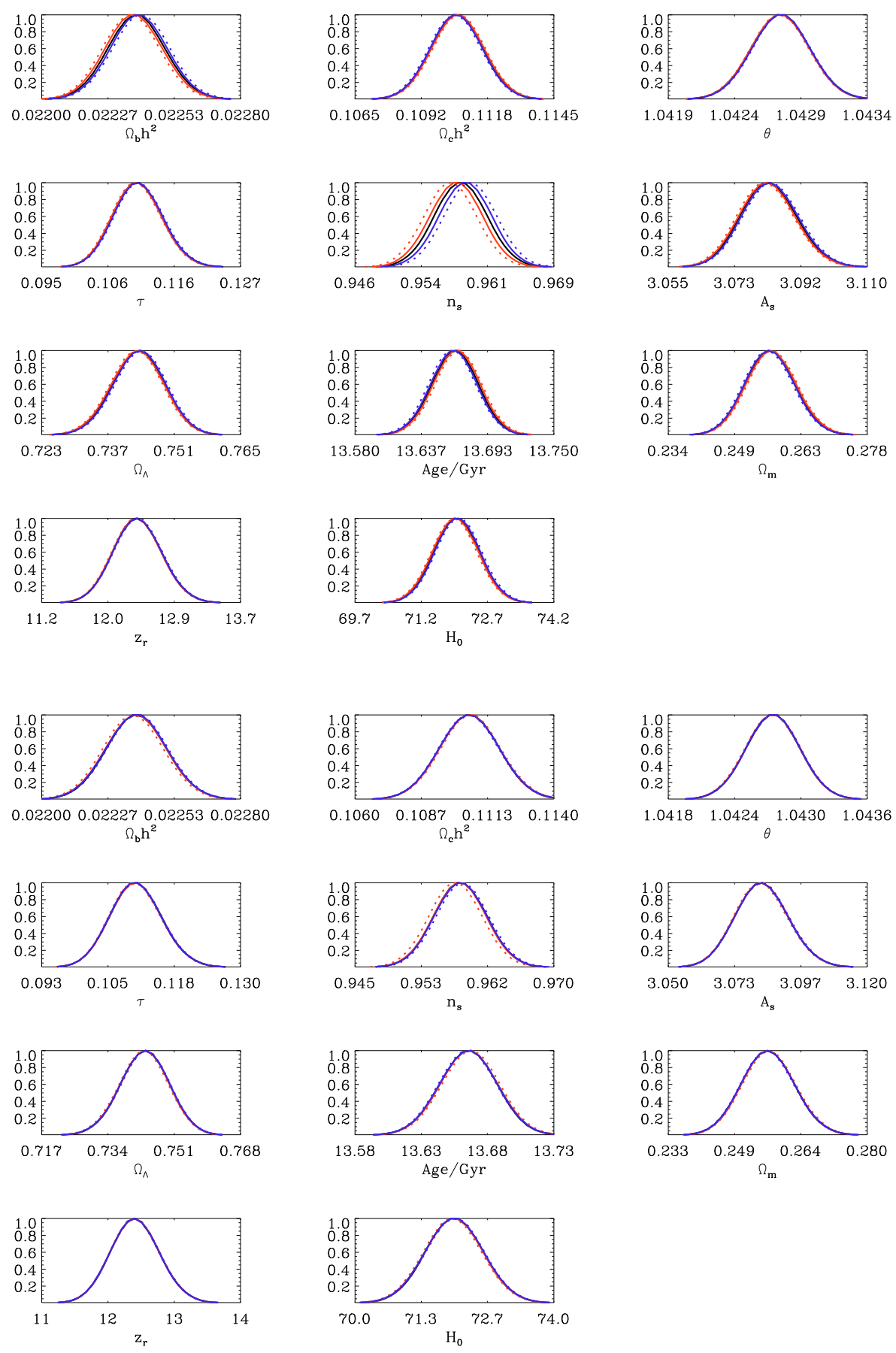
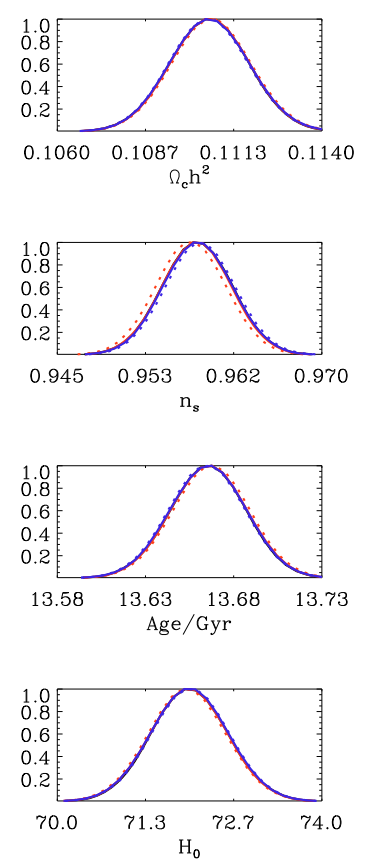
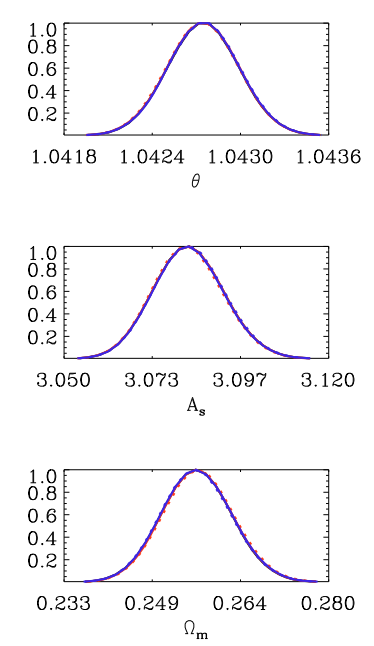
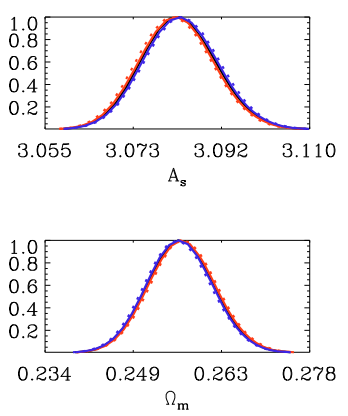

Fig. 19. Marginalized parameter constraints for Planck $143 \mathrm{GHz}$ with beam randomization MCBR: true beam (black), decreasing function for destriped data (red), increasing function for destriped data (blue), mild deviation (solid line) and extreme deviation from the true beam (dotted line).

Fig. 20. Marginalized parameter constraints for Planck $217 \mathrm{GHz}$ with beam randomization MCBR: true beam (black), decreasing function for destriped data (red), increasing function for destriped data (blue), mild deviation (solid line) and extreme deviation from the true beam (dotted line).

the specific systematic uncertainty. Furthermore it accounts accurately for the shape of the beam as it makes use of beam uncertainty templates for such beams, hence there is no need for simplified a priori assumptions on their shapes. Finally MCBR can be generalized and used to propagate other systematic uncertainties, as long as a set of templates of such systematics is provided.

From the study presented here on propagating the beam measurement errors to parameter estimation via the new MCBR method for Planck and for a future experiment, we conclude:

- Removal of $1 / f$ noise residuals, by destriping or other techniques, is quite important.
- The main impact of beam uncertainties is to widen the marginal distributions of some parameters (most notably $n_{\mathrm{s}}$ ).

- Assuming as extreme beam deviation in the simulated data results in a biased estimation of some parameters (mainly of $n_{\mathrm{s}}$ ) due to incomplete marginalization.

- The parameters more noticeably impacted by beam uncertainties are: $n_{\mathrm{s}}, \Omega_{\mathrm{b}} h^{2}$ and $A_{\mathrm{s}}$

These results demonstrate the relevance of applying destriping techniques on Planck data to remove $1 / f$ noise.

When the beam fitting is performed in destriped data the uncertainties on the beams for say $100 \mathrm{GHz}$ are at most of the order of $0.5 \%$ for $\ell=1500$ which translates into an increase of parameter uncertainties at most of the order of $0.1 \%$. Instead the 
G. Rocha et al.: MCBR: a study of the impact of PLANCK beam measurement errors on cosmological parameter estimation
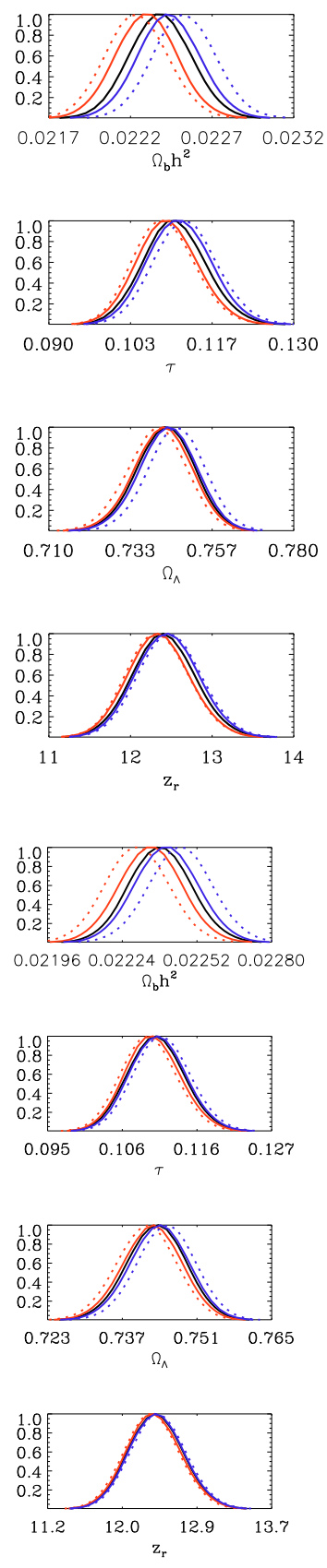
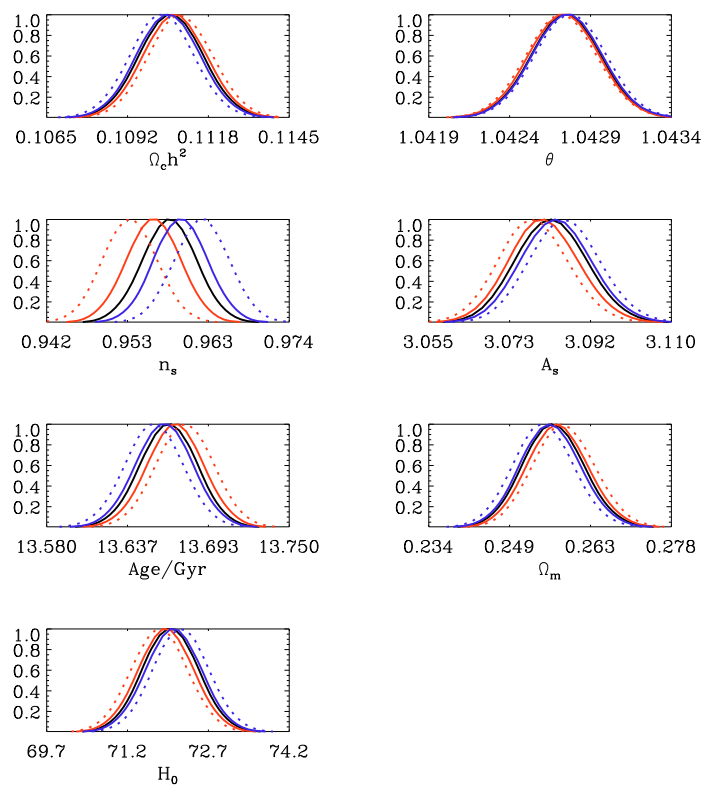
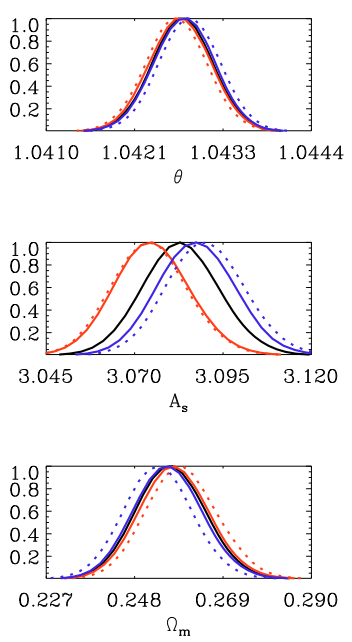

Fig. 22. Marginalized parameter constraints for Planck $143 \mathrm{GHz}$ with beam randomization MCBR: true beam (black), decreasing function for white $+1 / f$ noise (red), increasing function for white $+1 / f$ noise (blue), mild deviation (solid line) and extreme deviation from the true beam (dotted line).
Fig. 21. Marginalized parameter constraints for Planck $100 \mathrm{GHz}$ with beam randomization MCBR: true beam (black), decreasing function for white $+1 / f$ noise (red), increasing function for white $+1 / f$ noise (blue), mild deviation (solid line) and extreme deviation from the true beam (dotted line).

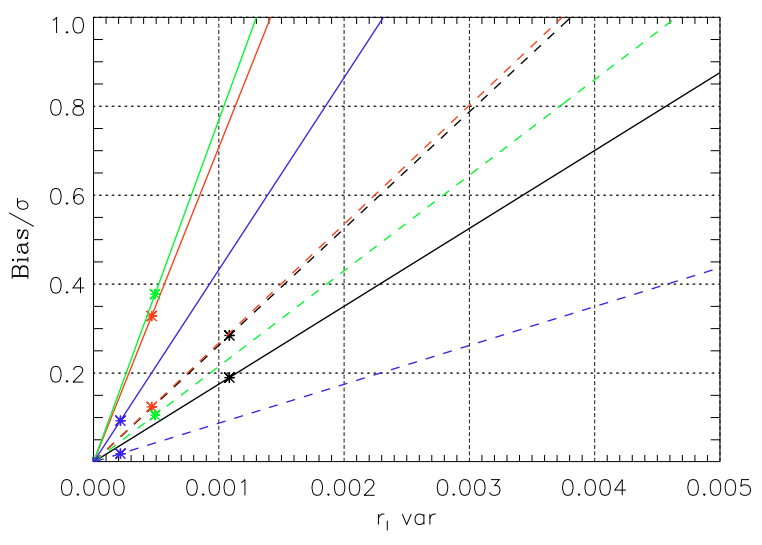

Fig. 23. Bias on $n_{\mathrm{s}}$ (solid line) and $A_{\mathrm{s}}$ (dotted line) in units of the error for the extreme beam functions, $r_{\ell}^{\text {ext }}$ for $\ell=1 / \sigma_{v}$ after beam randomization MCBR, fitted on destriped data. For $70 \mathrm{GHz}$ (black), $100 \mathrm{GHz}$ (red), $143 \mathrm{GHz}$ (green) and $217 \mathrm{GHz}$ (blue). 
Therefore we expect only a small impact of beam measurement errors on cosmological parameter estimation as long as the beam fitting is performed on destriped data.

Acknowledgements. G.R. is grateful to Jeffrey Jewell and Lloyd Knox for insightful discussions. L.P. acknowledges support by ASI contract I/016/07/0 "COFIS". K.M.H. receives support from NASA via JPL subcontract 1363745. We gratefully acknowledge support by the NASA Science Mission Directorate via the US Planck Project. The research described in this paper was partially carried out at the Jet propulsion Laboratory, California Institute of Technology, under a contract with NASA. Copyright 2009. All rights reserved.

\section{References}

Ashdown, M. A. J., Baccigalupi, C., Balbi, A., et al. 2007a, A\&A, 467, 761 Ashdown, M. A. J., Baccigalupi, C., Balbi, A., et al. 2007b, A\&A, 471, 361
Ashdown, M. A. J., Baccigalupi, C., Bartlett, J. G., et al. 2009, A\&A, 493, 753 Bock, J., Cooray, A., Hanany, S., et al. 2008, [arXiv: 0805.4207]

Bond, J. R., Jaffe, A. H., \& Knox, L. 2000, ApJ, 533, 19

Bridle, S. R., Crittenden, R., Melchiorri, A., et al. 2002, MNRAS, 335, 1193B Fendt, W. A., \& Wandelt, B. D. 2006, ApJ, 654, 2

Huffenberger, K. M., Crill, B. P., Lange, A. E., et al. 2010, A\&A, 510, A58

Lewis, A. 2005, Phys. Rev. D, 71, 083008

Lewis, A., \& Bridle, S. 2002, Phys. Rev. D, 66, 103511

Lewis, A., Challinor, A., \& Lasenby, A. 2000, ApJ, 538, 473

Planck Collaboration, Planck Blue Book 2005 [arXiv:astro-ph/0604069] Poutanen, T., CTP, et al. 2006, A\&A, 449, 1311

Rocha, G., Magueijo, J., Hobson, M., \& Lasenby, A. 2001, Phys. Rev. D, 64, 063512

Sandri, M., Bersanelli, M., Burigana, C., et al. 2002, in Experimental Cosmology at Millimetre Wavelengths, ed. M. de Petris, \& M. Gervasi, AIP Conf. Ser., 616,242

Spergel, D. N., et al. [WMAP Collaboration] 2003, ApJS, 148, 175

Yurchenko, V. B., Murphy, J. A., \& Lamarre, J.-M. 2004, Proc. SPIE 5487, ed. J. C. Mather, 542 